\title{
COMPETENCY II: CULTURAL AND HUMAN DIVERSITY
}

Considering the plethora of research literature in this domain, we had the daunting task of selecting a limited number of key articles that increase knowledge and contribute to improving practice. We first had to acknowledge that culture and human diversity are related in complex ways and impacted by historical legacies that influence policy, practice, and research. We concentrated on selecting "Cultural and Human Diversity" articles within the three categories assigned: classic, research, and practice. Knowing how important and integral "Cultural and Human Diversity" is to child and youth work, we wanted to ensure that our selections would further benefit the field.

Each editor was tasked with selecting and presenting articles in all three categories. We spent several weeks reviewing, discussing, and deliberating over articles until we were able to make our final selections in the three assigned categories as follows.

Classic: Cross, T. L. (1988). Services to minority populations: What does it mean to be a culturally competent professional?

Cross, T. L. (1988). Services to minority populations: Cultural competence continuum.

Research: Aisenberg, E. (2008). Evidence-based practice in mental health care to ethnic minority communities: Has its practice fallen short of its evidence?

Practice: McPhatter, A. R., \& Ganaway, T. L. (2003). Beyond the rhetoric: Strategies for implementing culturally effective practice with children, families, and communities.

\section{CLASSIC}

The first article, "What Does It Mean to be a Culturally Competent Professional?" offers a framework for understanding the skills and knowledge needed by service providers to become culturally competent. The article presents five crucial elements that must be mastered in order to achieve this goal: awareness and acceptance of differences, awareness of one's own cultural values, understanding of the dynamic of differences in the helping process, knowledge of the clients' culture, and adapting the practice skills and interventions to meet the clients' culture.

The second article by Cross, "Cultural Competence Continuum" provides organizations and individuals with a tool to utilize in assessing where they are in their journey of becoming culturally competent. Cross suggests that cultural competence is a set of congruent behaviors, attitudes, and polices that come together in a system, agency, or professional and enable that system, agency, or professional to work effectively in cross-cultural situations. Becoming culturally competent is a developmental process, and the learning is continuous. The developmental model presented in this article provides a framework that is set along a six-stage continuum beginning with cultural destructiveness and ending with cultural proficiency.

Both articles by Cross have been widely disseminated over the last 20 years in the field of human services. Many agencies and individuals have utilized the 
frameworks and guiding principles presented in these two articles to assist them in providing culturally competent services to children, youth, and families. These two articles provided the opportunity for agency staff, management, and boards to have a safe, open dialogue, and understanding about becoming cultural competent. The articles also pointed out how complex of an issue it is to become culturally competent and how important it is to institutionalize this practice throughout the whole organization. The editors feel that the issues, strategies, and frameworks presented in these two classic, interrelated articles are as relevant in 2011 as they were in 1988. This is evident by the fact that numerous systems (i.e., child welfare, mental health, juvenile justice, education, etc.) continue to base their approaches to cultural and linguistic competence on the strategies and framework offered in these articles.

\section{RESEARCH}

The research article, "Evidence-based Practice in Mental Health Care to Ethnic Minority Communities: Has its Practice Fallen Short of its Evidence?", provided an overview of the Evidence-Based Practice (EBPs) movement that grew out of the concern that many individuals were receiving ineffective services that were grounded in tradition and outdated training rather than scientific evidence of effectiveness. The article also expressed concern that the excessive attention given to the development and implementation of EBPs decreased focus on cultural difference within the service delivery system. EBPs tend to invalidate or leave out culturally specific interventions and traditional healing practices used in communities of color. EBPs are supported by documented evidence of effectiveness with certain populations and communities, and these interventions are equally effective and applicable across ethnic populations and communities. Nevertheless, there is little documented evidence that systematically validates this assumption. It is recommended that EBPs should be tested among diverse populations in their local community and treatment settings. This approach should also be developed in a bottom-up method that includes collaboration with diverse cultural and ethnic groups. These findings suggest that the current methodology for developing EBPs needs to be modified to include cultural and human diversity. We felt that this article provides vital information to practitioners that are required to use EBPs.

\section{PRACTICE}

In the practice-oriented article, "Strategies for Implementing Culturally Effective Practice with Children, Families, and Communities," cultural competence is expressed as the ability to transform knowledge and cultural awareness into health or psychosocial interventions that support and sustain healthy client-system functioning within the appropriate cultural context (McPhatter, 1997). McPhatter \& Ganaway, (2003) in"Beyond the Rhetoric: Strategies for Implementing Culturally Effective Practice with Children, Families, and Communities," present specific strategies that enhance cultural competence and effectiveness at the individual and multi- 
system levels that include work with the individual, among practitioners (interprofessionally), and within the organization interprofessional, middle management, and upper management levels. The framework for change is rooted in a five-stage model moving practitioners and agencies along a change continuum that includes the stages of precontemplation, contemplation, preparation, action, and maintenance. The authors explored barriers to culturally competent practice and multilevel strategies that work within child welfare agencies. They contend that"cultural competence as a goal becomes reality when those who set policy and control resources commit to outcome-based organizational change process."This article was selected because it moves practitioners and agencies from the "talk" of cultural competence to the "walk" of implementing culturally effective practice with children, families, and communities.

In conclusion, respect for cultural and human diversity is essential to child and youth work and provides a framework for regarding how we value children and youth within the larger context of society. The compiling of these articles has been an undeniably valuable experience for the editors, and we hope that future readers and in particular child and youth workers engage in the process of reflection, research, and rethinking our practice in ways that will improve outcomes for youth and children.

The editors have also compiled an additional reading list of 12 articles that would serve to be beneficial to youth workers in their quest to increase their understanding of the Cultural and Human Diversity Domain. In order to become cultural competent in our work we must be willing to embrace all cultures and human differences. The editor's definition of culture and human diversity is broad and represented in the various articles from rural issues to sexual orientation. We realize that the list is not complete, but we offer it as a starting point to further the learning which will result in improved practices for everyone.

\section{References}

McPhatter, A. (1997). Cultural competence in child welfare: What is it? How do we achieve if? What happens without it? Child Welfare, 76, 255-278.

\section{Final Referenced Selections}

Aisenberg, E. (2008). Evidence-based practice in mental health care to ethnic minority communities: Has its practice fallen short of its evidence? Social Work 53(4), pp. 297-306.

Cross, T. L. (1988). Services to minority populations: Cultural competence continuum. Focal Point. Portland, Oregan: Portland State University. 
Cross, T. L. (1988). Services to minority populations: What does it mean to be a culturally competent professional? Focal Point. Portland, Oregan: Portland State University.

McPhatter, A. R., \& Ganaway, T. L. (2003). Beyond the rhetoric: Strategies for implementing culturally effective practice with children, families, and communities. In J. J.Velazquez, A. R. McPhatter, \& K. Yang, Child Welfare Journal of Policy, Practice, and Program: Special Issue Perspectives on Cultural Competence (103-124).

\section{Cultural and Human Diversity Domain Editors}

Lloyd Bullard, $M E d$

LB International Consulting, LLC

Peter R. Correia III, MSW

The University of Oklahoma OUTREACH

National Resource Center for Youth Services

Deborah Green, MSW, MAC, CDVC-III, CCJS, BEI-1

Texas Department of Family Protective Services, Child Protective Services 